\title{
BOUNDARY BEHAVIOR OF GREEN POTENTIALS
}

\author{
DANIEL H. LUECKING ${ }^{1}$
}

Abstract. A Green potential on the unit disk $\{|z|<1\}$ is a function $u(z)$ of the form

$$
u(z)=\int \log \left|\frac{1-\bar{w} z}{z-w}\right| d \alpha(w)
$$

where $\alpha$ is a positive measure such that $\int(1-|w|) d \alpha(w)$ is finite. In this note I give a necessary and sufficient condition on a relatively closed subset $F$ of the unit disk in order that, for all such $u(z)$,

$$
\liminf _{F \ni: \rightarrow 1}(1-|z|) u(z)=0 .
$$

The condition is that the hyperbolic capacity of the portion of $F$ in arbitrarily small neighborhoods of 1 is bounded away from zero.

1. Introduction. A theorem due to $\mathrm{M}$. Heins [1, Theorem 6.1] states that, for a Blaschke product $B$ on the unit disk $U=\{z \in \mathbf{C}:|z|<1\}$ and any $e^{i \theta} \in \partial U$,

$$
\liminf _{r \rightarrow 1}(1-r) \log \left(\frac{1}{\left|B\left(r e^{i \theta}\right)\right|}\right)=0 .
$$

A rather simpler proof was given in [3] by J. H. Shapiro and A. L. Shields. Then in [2] W. C. Nestlerode and M. Stoll extended (1.1) to the unit polydisk in $\mathbf{C}^{n}$, with the function $\log (1 /|B|)$ replaced by an arbitrary positive $n$-superharmonic function whose greatest $n$-harmonic minorant is zero. In a later paper [4] Stoll took up the question of boundary approach more general than the radial approach in (1.1). In particular, he showed the following.

Theorem A. Let $\alpha$ be a positive measure on $U$ such that $\int(1-|w|) d \alpha(w)<+\infty$ and let

$$
u(z)=\int_{U} \log \left|\frac{1-\bar{w} z}{z-w}\right| d \alpha(w)
$$

If $\gamma[0,1) \rightarrow U$ is any curve satisfying $\lim _{t \rightarrow 1} \gamma(t)=1$, then

$$
\liminf _{t \rightarrow 1}(1-|\gamma(t)|) u\left(\gamma(t) e^{i \theta}\right)=0
$$

for any $e^{i \theta} \in \partial U$.

Received by the editors November 5, 1984 and, in revised form, April 22, 1985.

1980 Mathematics Subject Classification. Primary 31A20, 31 A15.

Key words and phrases. Green potentials, hyperbolic capacity.

${ }^{1}$ Supported in part by a National Science Foundation grant. 
If $B$ is a Blaschke product with zero set $\left\{a_{n}\right\}$, then $\log (1 /|B|)$ has the form (1.2) for the measure $\alpha$ which is concentrated on $\left\{a_{n}\right\}$ and assigns to each $a_{n}$ its multiplicity as a zero of $B$. The finiteness of $\int(1-|w|) d \alpha(w)$ is just the Blaschke condition on the zeros of $B$. Thus Theorem $\mathrm{A}$ is a generalization of Hein's result. Of course, functions of the form (1.2) are exactly the positive superharmonic functions on $U$ with zero for a greatest harmonic minorant, so the results of Nestlerode and Stoll fit in the same context.

In this article I consider even more general boundary approach than along a curve and, in fact, obtain necessary and sufficient conditions on a set, so that the analogue of Theorem A is valid for approach along rotations of that set. First I shall establish some terminology. A function $u(z)$ of the form (1.2) is called a Green potential. The condition $\int(1-|w|) d \alpha(w)<+\infty$ is equivalent to the condition $u \not \equiv+\infty$. This is because $1-|w| \leqslant C_{z} \log |(1-\bar{w} z) /(z-w)|$, so if $u$ is finite at even one point, then $1-|w| \in L^{1}(\alpha)$. Conversely, if $1-|w| \in L^{1}(\alpha)$, then Fubini's Theorem establishes that $u(z)$ is integrable with respect to area over $\{|z|<r\}$ if $r<1$. I use $\eta(E)$ to denote the hyperbolic capacity of the set $E \subset U$. This notion will be defined in the next section, where its necessary properties as well as its relationship to ordinary logarithmic capacity are presented. The main result is the following.

THEOREM 1. Let $F$ be a relatively closed subset of $U$. Then a necessary and sufficient condition on $F$ in order that

$$
\liminf _{F \ni z \rightarrow 1}(1-|z|) u(z)=0 \text { for every Green potential } u \not \equiv+\infty
$$

is that

$$
\lim _{\varepsilon \rightarrow 0} \eta(F \cap\{z:|z-1|<\varepsilon\})>0
$$

A word about $(\mathrm{B})$ is in order. Let $D(1, \varepsilon) \equiv\{z:|z-1|<\varepsilon\}$. It turns out that $0 \leqslant \eta(E) \leqslant 1$ for all sets $E$, and that (B) occurs if and only if $\eta(F \cap D(1, \varepsilon))=1$ for every $\varepsilon>0$. That is, the limit in (B) is always either 0 or 1 .

If $1 \notin \bar{F}$, the closure of $F$, then (B) is not satisfied and (A) is meaningless (which we interpret as being not satisfied). So it is not with any loss of generality that I will always assume $1 \in \bar{F}$. The implication $(\mathrm{A}) \Rightarrow(\mathrm{B})$ will be proved by showing something which is only apparently stronger: If $(B)$ is not satisfied, then there is a Green potential $u \neq \equiv+\infty$ such that $\liminf _{F \ni z \rightarrow 1}(1-|z|) u(z)=+\infty$. The measure $\alpha$ corresponding to this $u$ may be chosen with $F$ as its support.

I maintain the convention that $u$ denotes a Green potential, $u \neq \equiv+\infty$, and $\alpha$ denotes the corresponding measure with $\int(1-|w|) d \alpha(w)<+\infty$. All measures are positive Borel measures and the auxiliary measures $\mu, \mu_{\eta}$ that occur later will always be finite.

I would like to thank M. Stoll for posing the question answered here. I wish to acknowledge the hospitality of the Department of Mathematics at the University of Tennessee, where much of the research was accomplished. 
2. Hyperbolic capacity. The concept of hyperbolic capacity is similar to that of logarithmic capacity. It is defined in the same manner and differs only in that the Green function replaces the logarithmic kernel. For the unit disk the Green function is

$$
g(z, w) \equiv \log (|1-\bar{w} z| /|z-w|) \equiv \log (1 / \rho(z, w)),
$$

where $\rho(z, w)$ is the usual pseudohyperbolic distance $|z-w| /|1-\bar{w} z|$.

For an arbitrary Borel set $E \subset U$ define

$$
V(E)=\inf \iint g(z, w) d \mu(w) d \mu(z)
$$

where the infimum is taken over all positive measures $\mu$ of total mass 1 that are supported on compact subsets of $E$. The (inner) hyperbolic capacity of $E$ is defined to be $\exp [-V(E)]$ and is denoted $\eta(E)$. Clearly $0 \leqslant V(E) \leqslant+\infty$, so $0 \leqslant \eta(E) \leqslant 1$.

I will collect in this section the properties of hyperbolic capacity necessary for the proof of the theorem. Some of these can be found in M. Tsuji's book [5]. For some I will refer to the corresponding result for ordinary (logarithmic) capacity, with the understanding that the proof is almost identical for hyperbolic (or almost any other) capacity. Finally, I have been unable to find the proofs of one or two properties in the literature, so I have included them.

It is clear that

$$
\text { if } E \subset F, \text { then } \eta(E) \leqslant \eta(F) .
$$

One property we will need to obtain the $(\mathrm{A}) \Rightarrow(\mathrm{B})$ implication is the existence of an equilibrium distribution.

If $E$ is a compact subset of $U$, then there is a positive measure $\mu$ on $\partial E$, of total mass 1 , such that the infimum defining $V(E)$ is attained. The function $v(z)=$ $\int g(z, w) d \mu(w)$ is everywhere bounded by $V(E)$, is equal to $V(E)$ on $E$ except for a set with capacity zero, and is identically $V(E)$ on the interior of $E$. If $V(E)<+\infty$, then the measure $\mu$ gives no mass to any set of capacity zero.

This is a combination of results [5, pp. 75-77, 95]. The next property is that of outer regularity.

If $\left\{G_{n}\right\}$ is a sequence of open sets with $\bar{G}_{n+1} \subset G_{n}$ for each $n$, and if $\cap G_{n}=E$, a compact subset of $U$, then $\lim _{n \rightarrow \infty} \eta\left(G_{n}\right)$ $=\eta(E)$.

This can be found (for ordinary capacity) in [5, Theorem III.9].

A slight generalization of (2.2) will be required.

The conclusions of (2.2) are unchanged if $E$ is only assumed to be relatively closed in $U$, provided $V(E)>0$.

Proof. Let $E_{n}=E \cap \bar{D}(0,1-1 / n)$ so that $E_{n}$ is compact, $V_{n} \equiv V\left(E_{n}\right) \geqslant V \equiv$ $V(E)$, and $V_{n} \rightarrow V$. If $V=+\infty$, then all $V_{n}=+\infty$. Take the measures $\mu_{n}$ promised by (2.2) and let $\mu=\sum 2^{-n} \mu_{n}$. 
So assume $0<V<+\infty$, and let $\mu_{n}$ be the equilibrium distribution for $E_{n}$. Let $\mu$ be the weak* limit of the $\mu_{n}$ (as elements of $C_{0}(E)^{*}$ ). Clearly $\mu \geqslant 0$ and $\mu(E) \leqslant 1$. I claim that actually $\mu(E)=1$. To see this, let $k \leqslant n$ and observe that

$$
\begin{aligned}
V & \leqslant V_{n}=\int_{E_{k}} \int_{E_{n}} g(z, w) d \mu_{n}(w) d \mu_{k}(z) \\
& =\int_{E_{n}} \int_{E_{k}} g(z, w) d \mu_{k}(z) d \mu_{n}(w) \\
& =\int_{E_{k}} v_{k}(w) d \mu_{n}(w) \leqslant V_{k} \mu_{n}\left(F_{k}\right)+\varepsilon V,
\end{aligned}
$$

where $F_{k}=\left\{w \in E: \rho(z, w) \leqslant e^{-\varepsilon V}\right.$ for some $\left.z \in E_{k}\right\}$, because $v_{k} \leqslant \varepsilon V$ on $U \backslash F_{k}$. Fix $k$, so that $V \leqslant V_{k} \leqslant(1+\varepsilon) V$, to obtain $\mu_{n}\left(F_{k}\right) \geqslant(1-\varepsilon) /(1+\varepsilon)$. Letting $n \rightarrow \infty$, we see that $\mu\left(F_{k}\right)$ is arbitrarily close to 1 , and so $\mu(E)=1$. It remains to show that $\mu$ has the required properties. Define $g_{m}(z, w)=\min \{m, g(z, w)\}$. Then

$$
\begin{aligned}
v(z) & \equiv \int g(z, w) d \mu(w)=\lim _{m \rightarrow \infty} \int g_{m}(z, w) d \mu(w) \\
& =\lim _{m \rightarrow \infty} \lim _{n \rightarrow \infty} \int g_{m}(z, w) d \mu_{n}(w) \leqslant \lim _{n \rightarrow \infty} \int g(z, w) d \mu_{n}(w) \\
& \leqslant \lim _{n \rightarrow \infty} V_{n}=V .
\end{aligned}
$$

Suppose $V(z)<V-\varepsilon$ on a compact set $F \subset E$ with positive capacity. Then there is an equilibrium distribution $\mu^{\prime}$ on $F$. Letting $\mu^{\prime \prime}=\left(\mu+\delta \mu^{\prime}\right) /(1+\delta)$ leads to the following estimates:

$$
\begin{aligned}
V & \leqslant \iint g(z, w) d \mu^{\prime \prime}(z) d \mu^{\prime \prime}(w) \\
& \leqslant \frac{V+2(V-\varepsilon) \delta+\delta^{2} V(F)}{1+2 \delta+\delta^{2}}=V-\frac{\delta(\varepsilon-\delta(V(F)-V))}{1+2 \delta+\delta^{2}} \\
& <V \quad \text { for small enough } \delta,
\end{aligned}
$$

a contradiction.

Because each of the measures $\mu_{n}$ is supported on $\partial E_{n}$, it follows that $\mu$ is concentrated on $\partial E \cap U$. It is automatic that $\mu$ cannot give any mass to a set of zero capacity. For if $K$ is compact with $\eta(K)=0$ and $\mu(K)>0$, then

$$
+\infty=V(K) \leqslant \int_{K} \int_{K} \frac{g(z, w)}{\mu(K)^{2}} d \mu(z) d \mu(w) \leqslant \frac{V(E)}{\mu(K)^{2}}<+\infty,
$$

a contradiction.

Finally, if $z_{0}$ is an interior point of $E$, then for large $k$ and $n, g_{k}\left(z_{0}, w\right)=g\left(z_{0}, w\right)$ a.e. with respect to $\mu$ and $\mu_{n}$. Thus

$$
\begin{aligned}
\int g\left(z_{0}, w\right) d \mu(w) & =\int g_{k}\left(z_{0}, w\right) d \mu(w)=\lim _{n} \int g_{k}\left(z_{0}, w\right) d \mu_{n}(w) \\
& =\lim _{n} \int g\left(z_{0}, w\right) d \mu_{n}=\lim V_{n}=V .
\end{aligned}
$$


The discussion following Theorem 1 relies for its verification on the following result.

$$
\begin{aligned}
& \text { If } E \text { contains the union of compact sets } F_{n} \text { such that } \inf \{|z| \text { : } \\
& \left.z \in F_{n}\right\} \rightarrow 1 \text {, and if } \eta\left(F_{n}\right)>r>0 \text {, then } \eta(E)=1 \text {. }
\end{aligned}
$$

Proof. It need only be shown that for any $\delta>0$ there is a measure on $E$ of total mass 1 so that $\iint g(z, w) d \mu(z) d \mu(w)<\delta$. Given any sequence $s_{k} \rightarrow 1-$, it is possible to select a subsequence $F_{k}^{\prime}$ of the compact sets $F_{n}$ with $\inf \{\rho(z, w)$ : $\left.z \in F_{k+1}^{\prime}, w \in \bigcup_{j=1}^{k} F_{j}^{\prime}\right\}>s_{k}$.

Let $\mu_{k}$ be the equilibrium distribution for $F_{k}^{\prime}$. Let $\mu^{N}=N^{-1}\left(\mu_{1}+\cdots+\mu_{N}\right)$ and consider

$$
\begin{aligned}
V(E) & \leqslant \iint g(z, w) d \mu^{N}(z) d \mu^{N}(x) \\
& =\frac{1}{N^{2}} \sum_{i, j} \iint \log \frac{1}{\rho(z, w)} d \mu_{i}(z) d \mu_{j}(w) \\
& \leqslant \frac{\log (1 / r)}{N}+\sum_{i<j} \log \left(\frac{1}{s_{j}}\right) \\
& \leqslant \frac{\log (1 / r)}{N}+\sum_{j=1}^{N} j \log \left(\frac{1}{s_{j}}\right) \leqslant \delta
\end{aligned}
$$

if $N$ and $\left\{s_{j}\right\}$ are suitably chosen.

If $\eta(F \cap D(1, \varepsilon))>r>0$ for every $\varepsilon>0$ as in (B), then there exists a compact set $F_{1} \subseteq F \cap D\left(1, \varepsilon_{1}\right)$ with $\eta\left(F_{1}\right)>r$. Choose $\varepsilon_{2}$ so that $F_{1}$ is disjoint from $D\left(1, \varepsilon_{2}\right)$ and find $F_{2} \subseteq F \cap D\left(1, \varepsilon_{2}\right)$ with $\eta\left(F_{2}\right)>r$. Continuing in this manner produces a sequence of sets in $F \cap D\left(1, \varepsilon_{1}\right)$ satisfying the hypothesis of (2.5). Thus $\eta\left(F \cap D\left(1, \varepsilon_{1}\right)\right)=1$. Since $\varepsilon_{1}$ is arbitrary, we get $\lim _{\varepsilon \rightarrow 0} \eta(F \cap D(1, \varepsilon))=1$, provided (B) holds.

It is nearly obvious that hyperbolic capacity is invariant under Moebius transformations of the disk. It is easily established, for subsets $E$ of $\{z:|z|<1 / 2\}$, that hyperbolic capacity $\eta(E)$ is equivalent to logarithmic capacity $\gamma(E)$. That is, there is a constant $C$ (equal to $4 / 3$ actually) such that $\gamma(E) / C \leqslant \eta(E) \leqslant C \gamma(E)$. Thus, the capacity $\eta(E \cap\{z: \rho(z, a) \leqslant 1 / 2\})$ can be estimated by estimating $\gamma\left(M_{a}(E) \cap\{|z| \leqslant 1 / 2\}\right)$ where $M_{a}(z)=(z-a) /(1-\bar{a} z)$. If $E$ is a curve (as in Theorem A) and $a \in E$, then $M_{a}(E) \cap\{|z| \leqslant 1 / 2\}$ contains a continuum of diameter at least 1/2. By Theorems III.4 and III.5 of [5] it follows that there is a lower bound on the logarithmic capacity of this set. Thus Stoll's theorem is a special case of Theorem 1: A curve $E$ tending to 1 satisfies

$$
\eta(E \cap D(1, \varepsilon)) \geqslant \eta(E \cap\{z: \rho(z, a) \leqslant 1 / 2\}) \geqslant \delta>0,
$$

provided $a$ is close enough to 1 and $a \in E$. Since (B) is satisfied, (A) follows, which is the same as (1.3). 
3. The proof of Theorem 1. The first step in the proof is to reduce it to a statement about finite measures. This is the same procedure used in [4]. Let $S(a)=\{z$ : $\rho(z, a) \leqslant 1 / 2\}$.

Lemma. Let $\int(1-|w|) d \alpha(w)<+\infty$ and let $d \mu(w) \equiv(1-|w|) d \alpha(w)$. Then

$$
\begin{aligned}
& \lim _{|a| \rightarrow 1}(1-|a|) \int_{U \backslash S(a)} g(z, a) d \alpha(z)=0, \\
& \lim _{|a| \rightarrow 1} \int_{U \backslash S(a)} g(z, a) d \mu(z)=0, \\
& \lim _{n \rightarrow \infty}\left(1-\left|a_{n}\right|\right) \int_{S\left(a_{n}\right)} g\left(z, a_{n}\right) d \alpha(z)=0(+\infty) \quad \text { iff } \\
& \left.\lim _{n \rightarrow \infty} \int_{S\left(a_{n}\right)} g\left(z, a_{n}\right) d \mu(z)=0 \quad \text { (respectively, }+\infty\right) .
\end{aligned}
$$

Proof. For $z \notin S(a), g(z, a) \leqslant \log 2$, and

$$
g(z, a) \leqslant C\left(1-\rho(z, a)^{2}\right)=C\left(1-|a|^{2}\right)\left(1-|z|^{2}\right) /|1-\bar{a} z|^{2} .
$$

Thus the limit in (i) is dominated by

$$
\lim _{|a| \rightarrow 1} C \int \frac{\left(1-|a|^{2}\right)^{2}}{|1-\bar{a} z|^{2}} d \mu(z)
$$

which tends to zero by Lebesgue's theorem on bounded convergence. Similarly, the integrand $\chi_{U \backslash S(a)}(z) g(z, a)$ in (ii) is bounded by $\log 2$ and tends pointwise to zero as $|a| \rightarrow 1$, so again the limit is zero. Finally, (iii) follows because, on $S(a)$, $(1-|a|) /(1-|z|)$ is bounded above and away from zero, uniformly in $a$.

The proof of Theorem 1 will be accomplished by showing, with $d \mu(z)=$ $(1-|z|) d \alpha(z)$, that under hypothesis (B), $\lim _{n \rightarrow \infty} \int_{S\left(a_{n}\right)} g\left(z, a_{n}\right) d \mu(z)$ is zero for some sequence $a_{n} \in F, a_{n} \rightarrow 1$, and that if (B) fails then there is a finite measure $\mu$ such that $\int g(z, a) d \mu(z) \rightarrow+\infty$ as $a \rightarrow 1, a \in F$.

So assume (B). Then there exist compact sets $F_{n} \subset F \cap D\left(1, \varepsilon_{n}\right)$ such that the $F_{n}$ are disjoint, $\varepsilon_{n} \rightarrow 0$, and $\eta\left(F_{n}\right)>\delta>0$. Consider the function $h(\zeta)=$ $\int_{S(\zeta)} g(z, \zeta) d \mu(z)$. We wish to find a sequence $\left\{a_{n}\right\} \subset F, a_{n} \rightarrow 1$, such that $h\left(a_{n}\right) \rightarrow 0$. This is done by showing that $\int_{F_{n}} h(\zeta) d \mu_{n} \rightarrow 0$ for some positive measures $\mu_{n}$ on $F_{n}$ with $\mu_{n}\left(F_{n}\right)=1$. To see that this is so, observe that

$$
\int_{F_{n}} h(\zeta) d \mu_{n}(\zeta)=\int_{U} \int_{F_{n}} \chi_{S(z)}(\zeta) g(z, \zeta) d \mu_{n}(\zeta) d \mu(z)
$$

by Fubini's Theorem (and the fact that $\left.\chi_{S(\zeta)}(z)=\chi_{S(z)}(\zeta)\right)$. If $\mu_{n}$ are chosen to be the equilibrium distributions of $F_{n}$, then the inner integral is at most $\log (1 / \delta)$ and is zero for $z \notin F_{n}{ }^{*}=\left\{z: \rho(z, w) \leqslant 1 / 2\right.$ some $\left.w \in F_{n}\right\}$. Thus

$$
\int_{F_{n}} h(\zeta) d \mu_{n}(\zeta) \leqslant \log \left(\frac{1}{\delta}\right) \mu\left(F_{n}^{*}\right) \rightarrow 0
$$

because $F_{n}{ }^{*} \subset D\left(1,10 \varepsilon_{n}\right)$ and $\cap D\left(1,10 \varepsilon_{n}\right)=\varnothing$. 
To get the converse suppose (B) is not satisfied and suppose first that $\eta(F \cap \bar{D}(1, \varepsilon))=0$ for some $\varepsilon>0$. Simply let $\mu$ be the measure promised in (2.4) for $F \cap \bar{D}(1, \varepsilon)$. Then $\int g(z, w) d \mu(w)=+\infty$ on $F$ near 1 , so its limit is also $+\infty$.

Now assume $\eta(F \cap \bar{D}(1, \varepsilon))>0$ for all $\varepsilon>0$ and choose $\left\{\varepsilon_{n}\right\}$ decreasing to zero so fast that, with $E_{n}=F \cap \bar{D}\left(1, \varepsilon_{n}\right) \backslash D\left(1, \varepsilon_{n+1}\right)$ and $\delta_{n}=\eta\left(E_{n}\right)$, we have $\sum\left[\log \left(1 / \delta_{n}\right)\right]^{-1 / 2}<+\infty$. Let $\mu_{n}$ be the equilibrium distribution for $E_{n}$ (from 2.5), so that

$$
\int_{E_{n}} g(z, w) d \mu_{n}(w)=V_{n} \equiv \log \left(\frac{1}{\delta_{n}}\right)
$$

for $z \in E_{n}$, except for a set of capacity zero. By lower semicontinuity $\int_{E_{n}} g(z, w) d \mu_{n}(w)>V_{n} / 2$ on $E_{n} \backslash F_{n}$, where $F_{n}$ is a closed subset of $E_{n}$ with $\eta\left(F_{n}\right)=0$. Let $\mu_{n}^{\prime}$ be any positive measure on $F_{n}$ with $\mu_{n}^{\prime}\left(F_{n}\right)=1$ satisfying the conclusions of (2.4) with regard to $F_{n}$. Then, with $\mu \equiv \sum V_{n}^{-1 / 2}\left(\mu_{n}+\mu_{n}^{\prime}\right)$, we have $\int g(z, w) d \mu(w)>V_{n}^{1 / 2}$ on $E_{n}$. Thus, clearly, $\lim _{F \ni z \rightarrow 1} \int g(z, w) d \mu(w)=+\infty$.

If I had not promised to construct $\mu$ concentrated on $F$, then I would have simplified the proof of the converse by first replacing $F$ by a set $F^{\prime}$ with the same property $\left(\eta\left(F^{\prime} \cap D(1, \varepsilon)\right) \rightarrow 0\right.$ ), containing $F$ in its interior (making use of $(2.3)$ ). This would avoid the separate treatment of capacity zero sets.

If I were willing to assume that $\bar{F} \cap \partial U=1$, the proof would be further simplified by avoiding any need for (2.4). The $E_{n}$ would all be compact. There would be no change in the $(B) \Rightarrow(A)$ portion which, it seems to me, is about as simple as it can ever get.

Of course any rotation of a Green potential is also a Green potential, so (B) implies

$$
\liminf _{F \ni z \rightarrow 1}(1-|z|) u\left(z e^{i \theta}\right)=0
$$

for any Green potential. That is, the lower limit in an approach to $e^{i \theta}$ along the rotate $e^{i \theta} F$ is zero for any $e^{i \theta} \in \partial U$.

4. Comments. The possibility of obtaining similar results for Green potentials of arbitrary domains depends largely on the validity of an analogue of the lemma. Assume that $U$ is an open set in $\mathbf{R}^{n}$ which is nice enough that its Green function $g(x, y)$ satisfies $\lim _{y \rightarrow \partial U} g(x, y)=0$ for each $x \in U$. Let $\alpha$ be a positive measure on $U$, and suppose that its potential $u(x)$ is finite at some point, say $u(0)<+\infty$. The expression $1-|z|$ in the lemma and theorem may be replaced by $g(x, 0)$. The question then is whether $\liminf _{F \ni x \rightarrow x_{0}} g(x, 0) u(x)=0$ for a point $x_{0} \in \partial U$. An examination of the proof of the lemma shows that the definition of $S(a)$ was chosen only to obtain $g(z, a) \leqslant C$ on $U \backslash S(a)$. In the present generality it is convenient to take this as the definition: $S(a)=\{x \in U: g(x, a)>C\}$ for some convenient constant. The validity of the proof of the lemma rests on the fact that there is another constant $C_{0}$ such that

$$
1 / C_{0}<g(x, 0) / g(a, 0)<C_{0}
$$


whenever $x \in S(a)$ and $a$ is near $\partial U$. I do not know if this inequality can be shown for general domains. The necessary properties of hyperbolic capacity extend fairly routinely to this context.

One could also consider more general kernels, obtaining the associated capacities and potentials. If the kernel is nice enough, the appropriate generalizations of the theorem again rest on (4.1). Here is one example: Let $k(x, y)=|x-y|^{-1}$ in $\mathbf{R}^{3}$ with associated Newtonian capacity and Newtonian potential. Let $F$ be an unbounded closed set in $\mathbf{R}^{3}$. Then a necessary and sufficient condition for every potential $u(x)$ to satisfy

$$
\liminf _{F \ni x \rightarrow \infty} \frac{1}{|x|} u(x)=0
$$

is that $F$ have infinite capacity (equivalently, $\operatorname{cap}[F \cap\{x:|x|>R\}]>\delta>0$ for all $R$ ). Here $\operatorname{cap} E=1 / V$ and $V=\inf \left\{\iint|x-y|^{-1} d \mu(x) d \mu(y): \operatorname{supp} \mu \subset E, \mu \geqslant 0\right.$, $\mu(E)=1\}$.

\section{REFERENCES}

1. M. Heins, The minimum modulus of a bounded analytic function, Duke Math. J. 14 (1947), 179-215.

2. W. C. Nestlerode and M. Stoll, Radial limits of $n$-superharmonic functions in the polydisc, Trans. Amer. Math. Soc. 279 (1983), 691-703.

3. J. H. Shapiro and A. L. Shields, Unusual topological properties of the Nevanlinna class, Amer. J. Math. 97 (1976), 915-936.

4. M. Stoll, Boundary limits of Green potential in the unit disc, preprint.

5. M. Tsuji, Potential theory in modern function theory, Chelsea, New York, 1959.

Department of Mathematical Sciences, University of ArKansas, FayetTeville, arkansas 72701 